\title{
Диспозиционность, энтимема и знание ${ }^{1}$
}

Д. В. ЗАЙцЕВ

Все наше знание нужно нам в конечном счете для практического применения

E.K. Войшвилло

\begin{abstract}
After a short introduction, in the second section, I consider E. Voishvillo's approach to formal explication of dispositional predicates. The third section is a brief presentation of relevantly interpreted enthymematic implication. In the forth section I make an attempt to draw all-in-one and present a formalization of (enthymematic) reasoning with (dispositional) public announcements.
\end{abstract}

Keywords: dispositional predicate, enthymematic implication, public announcement logic

\section{1 Введение}

Рассматривая какие-либо логические результаты Е.К. Войшвилло, необходимо исходно иметь в виду специфическое «войшвиллианское» понимание логики, ее роли и места в познавательной практике. Неслучайно фундаментальный двухтомный учебник логики, предназначенный по замыслу его авторов Е.К. Войшвилло и М.Г. Дегтярева «для студентов философских факультетов и преподавателей логики», был назван «Логика как часть теории познания и научной методологии». Как уже на первых страницах замечают его авторы, «значение логической правильности мышления, подчеркнем еще раз, состоит в том, что она является необходимым условием гарантированного получения

${ }^{1}$ Работа частично поддержана РГНФ. Грант № 13-03-00461. 
истинных результатов в решении задач, возникающих в процессе познания» [2, с. 27]. Чуть ниже, характеризуя современный этап развития логики, они отмечают, что «впервые логика нашла на современном этапе ее развития также важные применения, непосредственно связанные с практической деятельностью» [2, с. 33].

Это не значит, что Е.К. Войшвилло отказывал логике в ее философском статусе. Как раз наоборот, именно подчеркивая философский характер логики как своеобразной философской пропедевтики, он отводил ей роль одновременно и «специальной» (самостоятельной) науки и части теории познания. В свою очередь, эта «двусторонность» логики означает, что «необходимо рассматривать логические понятия с точки зрения не только гносеологической, но и методологической их значимости» $[2$, c. 3]. В итоге в составе науки логики выделяются «наряду с формальной логикой ... такие разделы, как логическая семиотика (исследование языка как средства познания), а также методология (изучение общенаучных методов и приемов познания)» $[2$, c. 17].

Переходя теперь уже к самой методологии, или логике научного познания, Е.К. Войшвилло констатирует, что активное развитие этого раздела логики в последние годы (ХХ века) потребовало уточнения понятия логического следования. «При этом оказалось, что понятие логического следования необходимо как основа для выяснения не только ряда понятий самой формальной логики... но и ряда методологически важных общенаучных понятий, например научного объяснения и предсказания, закона науки и . . диспозиционного предиката. . . [1, с. 12]. Вот так, в полном согласии с развиваемой концепцией науки логики, Е.К. Войшвилло обосновывает методологическое значение релевантной логики и понятия логического следования в своей монографии [1]. Одним из важных практических приложений релевантной логики Е.К. Войшвилло считал формальную экспликацию диспозиционных предикатов. 


\section{2 Диспозиционные предикаты}

Проблема адекватного определения диспозиционных предикатов (то есть таких свойств предметов, которые проявляются при определенных обстоятельствах) имеет старую и славную историю. Одна из первых серьезных попыток формализации диспозиционных предикатов была предпринята Р. Карнапом и критически поддержана Б. фон Юхосом еще в 20-30 гг. ХХ в. История развития взглядов на эту проблему в западной философии науки подробно изложена в [7]. В русскоязычной литературе эта проблематика обсуждается в работах Е.К. Войшвилло, В.А. Смирнова, Я.В. Шрамко.

Очевидная трудность, которую необходимо было преодолеть исследователям диспозиционности, была связана с экстенсиональным характером используемой для этого классической логики высказываний. Так, например, линия исследований, берущая начало от Р. Карнапа и Б. фон Юхоса, основывалась на использовании для определения диспозиционных предикатов особых редукционных предложений импликативного вида, содержащих термины, репрезентирующие проверочную операцию (традиционно обозначается параметром Р) и способ проявления соответствующего свойства (параметр R). Например, для предиката «растворимость» проверочной операцией будет помещение вещества в воду, а способом проявления — реальное растворение вещества в изменившихся условиях. В стандартном карнаповском варианте определение диспозиционного предиката выглядело следующим образом:

$D(x) \Leftrightarrow_{D F} P(x) \supset R(x)$.

Традиционно критики обращают внимание на иррелевантное свойство данного определения, являющееся следствием парадоксального характера материальной импликации.

(ir1) Диспозиционное свойство должно быть приписано предмету, который не подвергался проверочной операции.

Однако если бы это был единственнй недостаток нерелевантного определения диспозиционности, то для его устранения достаточно было бы заменить в нем материальную импликацию на импликацию одной из систем паранепротиворечивой логики, не прибегая к релевантной импликации. С парадоксами матери- 
альной импликации связана еще одна нежелательная характеристика данного определения.

(ir2) Диспозичионное свойство должно быть приписано предмету, для которого свойство, обозначаемое параметром $R$, является неотделимым.

Например, можно было бы утверждать, что свойство «быть настоящим мужчиной» является диспозиционным, поскольку если субъект таковым обладает, то проявляется оно всегда и во всем.

Именно в этих (в первую очередь, в (ir1)) недостатках предложенного определения Е.К. Войшвилло и увидел возможность применения релевантной логики. Он выбирает в качестве стартового момента так называемую «схему Юхоса» и вносит в нее «релевантные» коррективы. Теперь определение диспозиционного предиката принимает следующий вид:

$D(x) \Leftrightarrow_{D F} \exists x(\varepsilon(x) \wedge((\varepsilon(x) \wedge P(x)) \rightarrow R(x)))$,

где $\varepsilon$ есть некоторое структурное свойство предмета, с необходимостью обусловливающее появление реакции $\mathrm{R}$, а импликация $\rightarrow$ представляет собобой импликацию системы $\mathbf{E}$.

Некоторые добавления и коррективы были внесены в эту схему Я. Шрамко в [5] и мной в [4].

Возвращение к старой дискуссии сегодня продиктовано двумя соображениями. Одно из них касается удивительного свойства определений Войшвилло-Шрамко (тире здесь вполне оправдано, поскольку различия между двумя упомянутыми определениями хоть и важны по существу, но синтаксически не столь значительны), которое в конце прошлого века осталось, насколько я могу судить, незамеченным нами. Второе, на мой взгляд, открывает некоторые новые возможности для использования релевантно эксплицированной диспозиционности в эпистемической логике.

\section{3 Диспозиционность и интуиционистская энтимема}

Второй том фундаментального проекта "Entailment: the logic of relevance and necessity" вышел в свет в 1992 г., то есть через четыре года после публикации книги Е.К. Войшвилло, за пять лет 
до появления монографии Я.В. Шрамко и за восемь до проведения конференции, на которой было представлено мое выступление, а имеющий отношение к теме данной статьи результат в первичной форме был предложен еще раньше - в 1953 г.! В открывающей второй том "Entailment" VI главе под названием "Теория следования" есть два параграфа, посвященные энтимеме. При этом энтимема и соответственно «энтимемическая импликация» рассматриваются в контексте системы Е с пропозициональными кванторами. Самое интересное, что авторы этого раздела, рассматривая возможность формальной экспликации энтимемы как особого вида условного высказывания «если А, то, при условии, что пропущенная посылка истинна, В», приходят к следующему определению, практически буквально повторяющему схему Юхоса в трактовке Е.К. Войшвилло,

$A \supset B \Leftrightarrow_{D F} \exists r(r \wedge((r \wedge A) \rightarrow B))$,

при этом $r$ - это та самая пропущенная посылка, которая должна, во-первых, быть истинна, а во-вторых, вместе с имеющейся посылкой, превращать энтимему в корректный силлогизм.

Однако и этим неожиданные результаты не исчерпываются. Обсуждая далее понятие правильного энтимемического рассуждения, авторы "Entailment" обнаруживают существенное сходство в предложенной выше трактовке энтимемической импликации и в канонической, восходящей к Гейтингу, интерпретации интуиционистской импликации. «Импликация $p \rightarrow q$ имеет место, если и только если мы располагаем конструкцией $r$, которая, будучи присоединена к любой конструкции, служащей доказательством $p$, автоматически обеспечивает конструкцию, являющуюся доказательством $q$. Другими словами, доказательство $p$ вместе с $r$ формирует доказательство $q »$ [8, p. 98]. В полном соответствии с содержательной интуицией оказывается, что

1) если принять предложенное выше определение импликации つ, то множество теорем $\mathbf{E}^{\forall \exists p}$, содержащих только символы энтимемической импликации, конъюнкции и дизъюнкции, совпадает с позитивным фрагментом системы интуиционистской логики $\mathbf{H}$; 
2) если дополнительно определить $\neg A$ как $A \supset \forall p p$, то система $\mathbf{H}$ содержится в системе $\mathbf{E}$ с пропозициональными кванторами!

Подводя оптимистический итог сказанному выше, авторы "Entailment" замечают, что корректные рассуждения могут быть представлены и описаны следующим образом: если мы в процессе рассуждения позволяем себе опускать (очевидно) истинные посылки, то мы рассуждаем интуиционистски; если же мы настолько аккуратны, что «выписываем» все посылки, то такое рассуждение описывается системой $\mathbf{E}$ релевантной логики $[6$, p. 54].

Применительно к проблеме формальной экспликации диспозиционных предикатов данный результат уже не выглядит столь оптимистичным. Получается, что поскольку импликативный фрагмент системы $\mathbf{H}$ содержит парадоксальную аксиому утверждения консеквента, иррелевантное свойство (ir2) схемы фон Юхоса сохраняется и для определения Е.К. Войшвилло! Последнее соображение, на мой взгляд, могло бы придать новый оборот старой дискуссии.

В уже упоминавшихся тезисах [4] я предлагал альтернативный вариант определения диспозиционного предиката. $\mathrm{C}$ некоторыми коррективами оно выглядит следующим образом:

$$
D(S) \Leftrightarrow_{D F} \exists x \exists P \exists R((P(x) \rightarrow R(x)) \rightarrow \exists \varepsilon(\varepsilon(x) \rightarrow S(x))) .
$$

Содержательно оно означает, что некоторое свойство $S$ является диспозиционным, если и только если для некоторого предмета существуют проверочная процедура и регистрируемая реакция такие, что если подвергнутый процедуре предмет обладает регистрируемым свойством, то существует структурная характеристика этого предмета, обусловливающая наличие данного (диспозиционного) свойства. Не касаясь здесь содержательных особенностей данного определения, отмечу лишь, что оно свободно от иррелевантностей обоих видов. 


\section{4 Диспозиционность и знание}

Диспозиционность как предмет философского исследования далеко не ограничивается идеями Венского кружка и проблемой формальной экспликации диспозиционных предикатов. Так, по мнению автора статьи «Диспозиции: онтологическая перспектива и коммуникативная аппликация», «что касается мировой, прежде всего западной, философской мысли, то диспозиционность в ней сегодня - один из самых эвристичных предметов исследования. И не только в логическом плане, коим отечественные авторы привыкли ограничиваться, а в самом что ни на есть философском - онтологическом (метафизическом) и гносеологическом» [3, с. 99]. В контексте данной статьи интересно остановиться на обращении к диспозиционности Г. Райла и тех перспективах для эпистемической логики, которые в связи с этим открываются.

Г. Райл в основном и наиболее важном своем произведении «Понятие сознания» ("The Concept of mind") использует понятие диспозиционности для характеристики высказываний о ментальных понятиях. Эта тема достаточно широко обсуждается в литературе, русский перевод «Понятия сознания» был издан в 1999 году и вполне доступен для чтения, поэтому ниже я позволю себе ограничиться только фрагментарным изложением тех идей Г. Райла, которые имеют отношение к теме. Итак, анализируя лингвистические выражения, описывающие ментальные сущности, Г. Райл отмечает, что их следует трактовать как обозначающие не события (в некотором онтологическом универсуме), а - преимущественно - диспозиции. «Сказать, что человек знает что-то или стремится стать кем-то, это не значит сказать, что он именно в данный момент нечто делает или подвергается какому-то воздействию, напротив, это означает, что он по мере необходимости способен совершить определенные действия, или что он имеет склонность делать и чувствовать определенные вещи в ситуациях определенного сорта» [10, р. 100]. При этом в трактовке диспозиционных терминов Г. Райл придерживается «импликативной» концепции в духе идей Р. Карнапа и Б. фон Юхоса. 
Отдельного упоминания заслуживает трактовка Г. Райлом таких терминов, как «знать» и «быть убежденным» (believe). Несмотря на все нюансы, связанные с различным употреблением этих терминов в разном контексте (например, различение «знания, что» и «знания, как»), предложения, содержащие их вхождения, могут естественным образом быть истолкованы как диспозиционные. Так, утверждение «Джон убежден, что Земля круглая» допускает естественную диспозиционную интерпретацию. Это означает, что оно может быть представлено как гипотетическое условное, законоподобное высказывание, истинность которого устанавливается не через соответствие какомулибо описываемому факту, а через удовлетворение определенных условий. Например: если Джон увидит исчезающий за горизонтом корабль, то он объяснит это шарообразностью Земли, а не внезапным кораблекрушением. Подобные условия многообразны и, в отличие от случая со «стандартными» диспозиционными предикатами типа «растворимость», не могут быть сведены к паре <проверочная операция, регистрируемое проявление $>$. Примером выражения такого неоднозначного диспозиционного свойства в обыденном языке является термин «хрупкость».

Возвращаясь к теме данной статьи, я хотел бы ниже предпринять попытку экспликации «объявленного знания» в терминах импликативно понимаемой диспозиционности. Логика публичных объявлений (Public Announcement Logic, или PAL) как самостоятельное направление в эпистемической логике появляется в 1989 году благодаря работам Дж. Плаза [9]. Подробное и обстоятельное изложение методов и результатов логики публичных объявлений в более широком контексте динамической эпистемической логики можно найти в монографии [11].С определенной долей условности ее проблематика может быть охарактеризована как исследование рассуждений, осуществляемых с использованием объявленной информации. Следующий ниже пример проясняет, какого типа рассуждения моделируются средствами PAL.

«У вас вся спина белая» упрощенная версия известного примера «Muddy children» 
Рассмотрим рассуждения двух преподавателей $\alpha$ и $\beta$, вернувшихся на кафедру после проведенных лекций. Третий участник ситуации сообщает им (делает публичное объявление) «По крайней мере у одного из вас спина в мелу».

Самый простой вариант рассуждений.

Рассуждение $\alpha$.

Посмотрев на $\beta$ и увидев, что его спина чистая, он заявляет «Я знаю, что моя спина в мелу».

Рассуждение $\beta$.

Даже не глядя на $\alpha$, на основании его объявления он заявляет «Тогда я знаю, что у меня спина чистая».

Преподаватель $\alpha$ пришел к заключению о том, что его спина испачкана, используя в качестве посылок, во-первых, дизъюнктивное объявление третьего участника беседы, и во-вторых, фактофиксирующее утверждение «спина $\beta$ не грязная». Схема рассуждения - Modus Tollendo Ponens. При этом преподаватель $\beta$ рассуждал следующим образом: «Известно, что по крайней мере у одного из нас спина грязная $(\alpha \vee \beta)$. Коллега $\alpha$, посмотрев на меня, заявил, что его спина - грязная (объявлено $\alpha$ ). Он мог это сделать, только увидев, что у меня спина чистая $(\neg \beta)$, поскольку из посылок $\alpha \vee \beta$ и $\neg \beta$ логически следует $\alpha$. Значит, моя спина - чистая $(\neg \beta)$. Очевидно, что в данном случае рассуждение не укладывается в стандартные схемы дедуктивных умозаключений.

Согласно сложившемуся подходу к формализации подобных рассуждений, публичное объявление эксплицируется с помощью оператора $[\alpha] \beta$, содержательно интерпретируемого как « $\beta$ имеет место после (правдивого) объявления $\alpha »$. Логика публичных объявлений строится как расширение эпистемической логики данным оператором со следующим условием истинности. Пусть стандартная эпистемическая модель - это тройка $\langle W, R, V\rangle$, тогда $|[\alpha] \beta|_{w}^{M} \Leftrightarrow|\alpha|_{w}^{M} \Rightarrow|\beta|_{w}^{M / \alpha}$, где модель $M / \alpha=\left\langle W^{*}, R^{*}, V^{*}\right\rangle$ представляет собой ограничение модели $M$ на те миры, в которых верна $\alpha$.

Если теперь обратиться к идее использования импликативного подхода в экспликации диспозиционных свойств как основы для формализации рассуждений с публичными объявлениями, 
то можно усмотреть некоторую содержательную связь между рассмотренными схемами и примерами. Так, вполне естественно, на мой взгляд, трактовать рассуждения на основе объявлений как энтимемические. В нашем примитивном примере преподаватель $\beta$ фактически восстанавливал энтимему. Первичный анализ литературы пока не позволил мне обнаружить работы, в которых PAL трактовалась бы в энтимемическом ключе, а такой подход, как мне представляется, мог бы быть интересным.

Естественно, внесение определенных изменений в импликативную схему определения диспозиционных свойств просто необходимо. Во-первых, теперь речь пойдет не о свойствах, а о положениях дел или ситуациях, которые могут быть объявлены, что предполагает использование пропозициональной логики. Во-вторых, роль структурного свойства, обусловливавшего появление реакции на стимул, теперь будет играть та необъявленная информация (скрытая посылка), которая позволяет рассуждающему субъекту делать умозаключение. В-третьих, в качестве возможного первого варианта формализации предлагается несколько по-другому эксплицировать публичные объявления. Пусть $[\alpha]_{\gamma}$ означает «объявлено $\alpha$ на основании $\gamma »$, где $\gamma$ - это некоторое исходное (общее) знание или сделанное ранее публичное объявление. Синтаксическое определение $[\alpha]_{\gamma}$ такоBO:

$[\alpha]_{\gamma} \Leftrightarrow{ }_{D F} \exists \beta(\beta \wedge((\beta \wedge \gamma) \rightarrow \alpha))$, где $\beta$ представляет известную только некоторым субъектам информацию (скрытую посылку).

Применительно к нашему примеру, рассуждение преподавателя $\beta$ может быть реконструировано следующим образом

1. $\alpha \vee \beta-$ исходная информация

2. $[\alpha]_{\alpha \vee \beta}$ - объявление преподавателя $\alpha$

3. $\exists \gamma(\gamma \wedge((\gamma \wedge(\alpha \vee \beta)) \rightarrow \alpha))$ из 2 .

4. $((\alpha \vee \beta) \wedge \neg \beta) \rightarrow \alpha-$ схема корректного перехода в релевантной логике при условии доказанности $\neg \beta$ и $\alpha \vee \beta$

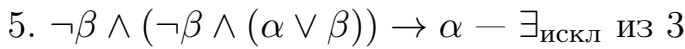

6. $\neg \beta-$ из 5 .

Естественно, предложенная формализация рассуждений весьма уязвима для критики и носит только предварительный характер. Тем не менее, представляется, что заявленный подход 
к экспликации рассуждений на основе публичных объявлений открывает новые интересные перспективы для дальнейших исследований.

\section{Литература}

[1] Войшвилло Е.К. Философско-методологические аспекты релевантной логики. М.: Изд-во МГУ, 1988.

[2] Войшвилло Е.К., Дегтярев М.Г. Логика как часть теории познания и научной методологии. М.: Наука, 1994.

[3] Гречко П.К. Диспозиции: онтологическая перспектива и коммуникативная аппликация // Вопросы философии. 2012. № 4. С. 99-110.

[4] Зайщев Д.В. К определению диспозиционных понятий // Материалы VI Общероссийской научной конференции «Современная логика: проблемы теории, истории и применения в науке». СПб.: Изд-во СПбГУ, 2000.

[5] Шрамко Я.В. Логическое следование и интуиционизм. Киев.: ВИПОЛ, 1997.

[6] Anderson A.R., Belnap N.D., Dunn J.M. Entailment: The Logic of Relevance and Necessity. Vol. II. Princeton, NJ.: Princeton University Press, 1992.

[7] Bird A. Dispositional Expressions // Routledge Companion to the Philosophy of Language / G. Russell and D . G. Fara (eds.). Routledge, 2012.

[8] Heyting A. Intuitionism. Amsterdam (Norht-Holland Publishing Company), 1971.

[9] Plaza J.A. Logics of public communications // Proceedings of the 4th International Symposium on Methodologies for Intelligent Systems / M.L. Emrich, M.S. Pfeifer, M. Hadzikadic, and Z.W. Ras (eds). 1989. P. 201-216.

[10] Ryle G. The Concept of Mind. Routledge. Taylor and Francis e-Library, 2009.

[11] van Ditmarsch H., van der Hoek W., Kooi B. Dynamic Epistemic Logic. Vol. 337 of Synthese Library Series. Springer Verlag, 2007.

\section{References (transliteration)}

[1] Vojshvillo E.K. Filosofsko-metodologicheskie aspekty relevantnoj logiki. M.: Izd-vo MGU, 1988.

[2] Vojshvillo E.K., Degtjarev M.G. Logika kak chast' teorii poznanija i nauchnoj metodologii. M.: Nauka, 1994. 
[3] Grechko P.K. Dispozicii: ontologicheskaja perspektiva i kommunikativnaja applikacija // Voprosy filosofii. 2012. № 4. S. 99-110.

[4] Zajcev D.V. K opredeleniju dispozicionnyh ponjatij // Materialy VI Obsherossijskoj nauchnoj konferencii "Sovremennaja logika: problemy teorii, istorii i primenenija v nauke". SPb.: Izd-vo SPbGU, 2000.

[5] Shramko Ja.V. Logicheskoe sledovanie i intuicionizm. Kiev.: VIPOL, 1997.

[6] Anderson A.R., Belnap N.D., Dunn J.M. Entailment: The Logic of Relevance and Necessity. Vol. II. Princeton, NJ.: Princeton University Press, 1992.

[7] Bird A. Dispositional Expressions // Routledge Companion to the Philosophy of Language / G. Russell and D . G. Fara (eds.). Routledge, 2012.

[8] Heyting A. Intuitionism. Amsterdam (Norht-Holland Publishing Company), 1971.

[9] Plaza J.A. Logics of public communications // Proceedings of the 4th International Symposium on Methodologies for Intelligent Systems / M.L. Emrich, M.S. Pfeifer, M. Hadzikadic, and Z.W. Ras (eds). 1989. P. 201-216.

[10] Ryle G. The Concept of Mind Routledge. Taylor and Francis e-Library, 2009.

[11] van Ditmarsch H., van der Hoek W., Kooi B. Dynamic Epistemic Logic. Volume 337 of Synthese Library Series. Springer Verlag, 2007. 